\title{
Philosophiques
}

\section{Bernard Lamy, La Rhétorique ou l'art de parler (éd. critique établie par Benoît Timmermans), Paris, PUF, (coll. " L’interrogation philosophique »), 1998, 598 p.}

\section{Lucie Desjardins}

Volume 27, numéro 1, printemps 2000

Le matérialisme contemporain

URI : https://id.erudit.org/iderudit/004914ar

DOI : https://doi.org/10.7202/004914ar

Aller au sommaire du numéro

Éditeur(s)

Société de philosophie du Québec

ISSN

0316-2923 (imprimé)

1492-1391 (numérique)

Découvrir la revue

Citer ce compte rendu

Desjardins, L. (2000). Compte rendu de [Bernard Lamy, La Rhétorique ou l'art de parler (éd. critique établie par Benoît Timmermans), Paris, PUF, (coll.

"L'interrogation philosophique »), 1998, 598 p.] Philosophiques, 27(1), 216-219. https://doi.org/10.7202/004914ar d'utilisation que vous pouvez consulter en ligne.

https://apropos.erudit.org/fr/usagers/politique-dutilisation/ 
Bernard Lamy, La Rhétorique ou l'art de parler (éd. critique établie par Benoît Timmermans). Paris, PUF, (coll. « L'interrogation philosophique »), 1998, $598 \mathrm{p}$.

L'édition de La R hétorique ou l'art de parler de Bernard Lamy que Benoît Timmermans a procurée au public en 1998 relève du tour de force. Paru pour la première fois en 1675 sous le titre D e l'art de parler, l'ouvrage sera maintes fois remanié et amplifié par son auteur à l'occasion des 29 éditions recensées dans lesquelles on passe progressivement de 288 pages à 444 pages. En présentant, de façon habile, le texte avec ses nombreuses variations, Timmermans fait plus que proposer les différentes strates du travail d'écriture. L'intérêt de ces multiples remaniements, écrit-il, est « qu'ils fournissent une suite de clichés représentatifs des états successifs de la problématique rhétorique, mais aussi philosophiques, qui animent les disciplines centrées sur le langage à la charnière des XVII ${ }^{e}$ et $X V I I{ }^{e}$ siècles $\gg(p .5)$.

L'ouvrage de Lamy entend se distinguer radicalement d'avec les rhétoriques que I'on retrouve habituellement à la même époque. Dès la préface, l'auteur les dénonce :

Les rhétoriques ordinaires sont faites de manière qu'elles sont presque entièrement inutiles. [...] Aussi il y a bien des gens qui font peu de cas de la rhétorique, ce qui n'arriverait pas si elle était bien traitée, c'est-à-dire si elle donnait effectivement la connaissance de ce qu'elle devrait enseigner; ; ce qu'il est bon de considérer ici (p. 14).

En contestant l'utilité des « rhétoriques ordinaires », Lamy effectue une intrusion remarquée dans le genre codifié du traité oratoire. Une tradition, déjà deux fois millénaire, voulait que le manuel de rhétorique se livrât à l'énumération des genres et des diverses parties de l'art oratoire avant de s'étendre sur certaines d'entre elles, en général sur l'inventio, puis, à un degré moindre, sur les deux suivantes, la dispositio et l'elocutio. C'est ce que fait, par exemple, R ené Bary dans sa R hétorique françoise (1659) : centrée sur l'inventio, celle-ci fournit à profusion des listes de lieux généraux, spécifiques, extrinsèques et intrinsèques. L'élève apprend alors à discourir comme en dialectique, en égrenant définition, énumération des parties, etc.

On ne retrouve pas, chez l'oratorien Lamy, de listes de lieux logiques : ceux-ci sont « dangereux pour les personnes qui ont peu de savoir, parce qu'elles se contentent de ces preuves qui se trouvent facilement, et qu'elles ne prennent pas la peine d'en chercher d'autres qui soient plus solides » (p. 453). L'art de parler ne sera donc qu'une partie de l'art de persuader, celle qui, dans l'ancienne rhétorique, avait pour nom elocutio. Et si on peut encore reconnaître bon nombre de notions empruntées à la rhétorique classique, c'est suivant un dispositif bien différent et avec des explications inédites.

Le premier livre traite de grammaire et de logique. À la suite de Port-R oyal, Lamy considère que « les paroles sont des signes qui représentent les choses qui se passent dans notre esprit », si bien qu'elles sont comme une « peinture de nos pensées, que la langue est le pinceau qui trace cette peinture et que les mots dont le discours est orné en sont les couleurs » (p. 35). La multitude des choses, le nombreinfini de manières dont l'esprit peut les envisager exige toutefois un lexique lui-même infini. Dans ces conditions, il est impossible d'imaginer une langue qui réponde parfaitement aux besoins de la conceptualisation en établissant une correspondance univoque entre les mots et les choses. A ussi le deuxième livre est-il entièrement consacré aux tropes et aux figures. Toutefois, il offre bien plus que cette sorte de répertoire des 
tropes et des figures illustré d'exemples que les rhétoriques se sont plu, au fil des siècles, à dresser avec une précision toujours accrue. Lamy tente plutôt d'exposer les raisons de l'apparition des tropes et des figures dans le discours. II en arrive alors à une question essentielle : celle des passions. Selon lui, le langage naturel est traversé par la passion, car il véhicule des idées par des mots qui ne traduisent pas forcément des idées claires et distinctes. En effet, le discours d'un sujet en proie à une ou plusieurs passions se distingue du discours de celui qui « parle sans émotion » :

Ainsi les paroles répondant à nos pensées, le discours d'un homme qui est ému ne peut être égal. Q uelquefois il est diffus, et il fait une peinture exacte des choses qui sont l'objet de sa passion : il dit la même chose en cent façons différentes. Une autre fois son discours est coupé, les expressions en sont tronquées; cent choses y sont dites à la fois : il est entrecoupé d'interrogations, d'exclamations ; il est interrompu par de fréquentes digressions ; il est diversifié par une infinité de tours particuliers et de manières de parler différentes. Ces tours et ces manières de parler sont tout aussi faciles à distinguer d'avec les façons de parler ordinaires que les traits d'un visage irritéd'avec ceux d'un visage calme (p. 182).

D ès lors, ces « tours particuliers » et ces « manières deparler différentes » ne sont que les signes d'une pensée bousculée par une expérience perceptive modifiée ou altérée par les passions. Aussi le langage de la passion n'est-il « pas libre », mais résulte d'un mouvement naturel, si bien que ce n'est plus le concept de signe, mais celui de figure qui servira désormais à se mouvoir dans cet univers. En effet,

les passions font que l'on considère les choses d'une autre manière que l'on ne le fait dans le repos et dans le calme de l'âme : elles grossissent les objets, elles y attachent l'esprit ; et font presque autant d'impression sur lui que les choses mêmes (p. 181).

C'est parce que les passions font que l'on considère les choses d'une autre manière qu'on ne le fait dans le « repos et dans le calme de l'âme » qu'elles modifient le rapport vécu au monde et, par conséquent, le discours. Reste à établir les modalités de ces modifications. Tout concourt alors à faire en sorte que la langue ne semble plus donner seulement accès à la pensée, mais à un regard particulier sur le monde que modulent et orientent les mouvements des passions.

Dans cette perspective, il ne saurait exister de passion sans une forme de représentation facilement reconnaissable et c'est dans des termes qui rappellent $L$ a confé rence sur l'expression générale et particulière de Charles Le Brun (1668) que Lamy observe tout aussi bien que le premier effet des passions tient à une altération qu'elles provoquent autant sur un visage vierge de toute marque d'agitation, que dans le discours dépourvu de figures d'un homme qu'aucune passion n'agite.

Comme on lit sur le visage d'un homme ce qui se passe dans son cœur ; que le feu de ses yeux, les rides de son front, le changement de couleur de son visage, sont les marques évidentes des mouvements extraordinaires de son âme; les tours particuliers de son discours; les manières de s'exprimer éloignées de celles que l'on garde dans la tranquillité, sont les signes et les caractères des agitations, dont son esprit est ému dans le temps qu'il parle (p. 181).

À partir de ce parallèle entre figures du discours et marques des passions qui se peignent naturellement sur le visage, Lamy aborde ensuite la question de la représen- 
tation des passions dans le discours. Plus que sur ces « manières de parler », l'attention se porte alors sur les raisons de ces manières. R este à établir les modalités du rapport entre ces figures du discours et les passions qu'elles représentent. S'il y a une correspondance entre figures du discours et passions de l'âme, ce rapport semble difficilement intégrable dans un code. Les figures qui sont les caractères discursifs des passions viennent rendre compte d'un regard particulier sur les objets du monde, regard particulier qui sert, à son tour, d'argument.

Dès lors, l'essentiel de la figure ne réside plus dans l'écart, la déviation de langage par laquelle elle se réalise, mais dans l'état affectif qu'elle traduit et qu'elle vise à communiquer. Encore une fois, L amy excède la rhétorique classique qui s'intéressait à l'elocutio en tant que technique ornementale et voyait dans « les fleurs de la rhétorique » un supplément destiné à agrémenter des œuvres dont la beauté devait d'abord consister dans la justesse d'ensemble. Parce qu'elles représentent les passions, les figures ne sont pas qu'un ornement du discours : elles sont « utiles et nécessaires » (p. 185). L'orateur peut s'en servir pour faire la peinture exacte d'une passion, pour convaincre qu'il est lui-même véritablement ému ou encore pour susciter une passion chez l'auditeur. Car les figures ne sont ici rien d'autre quela manière dont une passion modifie la perception et, par conséquent, le discours : elles sont un lieu qui permet à la passion de se trouver un langage propre et des signes authentiques à même de révéler une pensée conçue comme indissociable de la perception des choses. A ussi devront-elles s'appliquer à rendre le mouvement diffus de la pensée d'un individu livré à ses passions. En tenant compte de cette composante psychologique d'une perception des choses modifiée par la passion, Lamy s'emploiera, dans la suite du texte, à définir un certain nombre de figures susceptibles d'exprimer, mieux que d'autres, ce mouvement : I'exclamation, le doute, l'épanorthose, l'ellipse, l'aposiopèse, I'hyperbate, la paralipse, la répétition, la paronomase, le pléonasme, le synonyme, I'hypotypose, la description, la distribution, I'antithèse, la similitude, la comparaison, la suspension, la prosopopée, la sentence, l'épiphonème, l'interrogation, l'apostrophe, l'épistrophe, la prolepse, la communication, la confession, le consentement et la périphrase (p. 190-217). On remarquera que ces figures conviennent aussi pour des discours qui ne mettent pas en cause une passion.

En ce sens, le regard particulier que suppose la passion vient s'opposer, chez L amy, à la raison ou, pour mieux dire, à un rationalisme philosophique qui, au XVII siècle, vise à donner du monde une représentation qui se veut universelle. Les idées claires et distinctes de D escartes conduisent à une représentation univoque du monde, car rien de ce qu'on peut connaître par la raison dans le monde extérieur ne peut être différent de ce que représentent ces idées claires et distinctes, elles-mêmes garanties par un modèle logique qui en appelle, de manière ultime, à Dieu. Or, la question de la représentation des passions permet à Lamy d'introduire au cœur même de sa rhétorique, pourtant d'inspiration cartésienne, une théorie susceptible d'envisager la multiplicité des points de vue. Ce serait en effet se méprendre de croire que, si les figures marquent la passion, elles excluent toute forme de jugement : au contraire,

Le ton de la voix, et le tour qu'on prend fait connaître de quelle manière on regarde les choses dont on parle, le jugement qu'on en fait, et les mouvements dont on est animé à leur égard (p. 157).

Du moment que la passion nous fait considérer un objet, celui-ci perd son univocité et devient multiple. Les figures ne sont donc pas réglées sur une représentation 
du monde à vocation universelle, mais bien sur un regard et une perception particulière indissociable du mouvement de la passion.

Le troisième livre s'intéresse à l'autre versant du langage, à la substance phonique, c'est-à-dire à ce que Lamy, dans la métaphore qu'il emprunte à D escartes, appelle «le corps du discours ». Cette corporéité doit être analysée dans toute son étendue et avec le secours de l'ensemble des disciplines susceptible de donner à comprendre cette corporéité. Celivre va donc inclure, à côté des notions de phonétique générale, un vaste ensemble de questions relatives aux sciences, à la philosophie et à la poétique qui conduira l'auteur, dans le dernier livre, à aborder la question du style. Comme le discours est le « caractère de l'âme », il n'est pas étonnant qu'il y ait autant de différents styles qu'il y a de personnes qui parlent ou écrivent. D emême, les hommes qui vivent dans des zones climatiques différentes ont un style différent. Ici, la théorie des climats vise à rendre compte dela « couleur particulière » propreau rendu discursif dela perception. Au surplus, si Lamy reprend la tripartition reçue des styles (style sublime, style moyen et style simple), ce ne sera pas sans y ménager un espace à la passion :

lorsque les choses sont grandes, et que l'on ne peut les envisager sans ressentir quelque grand mouvement, le style qui les décrit doit être nécessairement animé, plein de mouvement, enrichi de Figures, de toutes sortes de M étaphores.

Si le sujet quel'on traite n'a rien d'extraordinaire, si on le peut considérer sans être touché de passion ; le style doit être simple (p. 378).

N égligée par les études sur la rhétorique qui ont longtemps considéré que la rhétorique française naissait avec Dumarsais et Fontanier, la R hétorique de L amy est tenue aujourd'hui, à juste titre, pour un texte essentiel Iorsqu'on s'intéresse à I'histoire des idées en général et à l'histoire de la rhétorique en particulier. Essentiel, car I'attention que Lamy porte à la question des passions dans le discours force à reconsidérer l'idée, trop souvent répandue depuis $M$ ichel Foucault, que le XVII ${ }^{\mathrm{e}}$ siècle tout entier est habité par le seul désir d'une langue qui viendrait refléter l'univocité des signes. Essentiel, parce que Lamy participe à un ample mouvement qui ira s'intensifiant au cours du siècle suivant et qui cherchera, par-delà l'enseignement de la rhétorique, à placer les phénomènes sensoriels et psychologiques au centre de la réflexion sur le langage et de la problématique esthétique. L'excellente édition de Benoît Timmermans fait plus que remettre au jour ce texte capital : elle présente surtout I'avantage de retracer les strates successives du travail de Lamy et, par là, permet de mieux comprendre le passage du «Grand Siècle » au « Siècle des Lumières ».

LUCIE DESJARDINS

Département d'études littéraires Université du Québec à Montréal

Jocelyn Benoist, Robert Brisart, Jacques English, Liminaires phénoménologiques : recherches sur le développement de la théorie de la signification de Husserl. Bruxelles, Publications des Facultés universitaires Saint-Louis, 1998, 281 p.

Cet ouvrage, dont le thème demeure encore inusité dans la littérature de langue française, tente d'investiguer à nouveaux frais la théorie de la signification de H usserl. En 